\title{
Sarcopenia: an emerging risk factor for non-alcoholic fatty liver disease
}

\author{
Yong-ho Lee ${ }^{1,2} \cdot$ Seung Up Kim ${ }^{1,3,4}$
}

Received: 15 July 2019 / Accepted: 1 November 2019 / Published online: 21 November 2019

(c) Asian Pacific Association for the Study of the Liver 2019

Abbreviations
$\begin{array}{ll}\text { NAFLD } & \text { Nonalcoholic fatty liver disease } \\ \text { NASH } & \text { Nonalcoholic steatohepatitis } \\ \text { CI } & \text { Confidence interval } \\ \text { OR } & \text { Odds ratio } \\ \text { EWGSOP } & \text { European Working Group on Sarcopenia in } \\ & \text { Older People }\end{array}$

Nonalcoholic fatty liver disease (NAFLD) has gradually become one of the most common chronic liver diseases worldwide. A recent US study reported that the increased liver-related mortality in that country was associated with the increased prevalence of NAFLD [1]. This is now being seen in other parts of the world, including Asia, due to the adoption of a westernized lifestyle, along with effective vaccination and potent therapeutic agents for chronic hepatitis $\mathrm{B}$ and $\mathrm{C}$ virus infection.

NAFLD encompasses many disease states, ranging from nonalcoholic fatty liver with no evidence of inflammation or hepatocellular injury, which is considered a benign condition, to nonalcoholic steatohepatitis (NASH), which can progress to liver cirrhosis, liver failure, and hepatocellular carcinoma [2]. Therefore, early identification of and intervention for NAFLD in patients who are at high risk for disease progression is required, both for medical and economic reasons.

Seung Up Kim

ksukorea@yuhs.ac

1 Department of Internal Medicine, Yonsei University College of Medicine, Yonsei-ro 50, Seodaemun-gu, Seoul, Republic of Korea

2 Institute of Endocrine Research, Yonsei University College of Medicine, Seoul, Republic of Korea

3 Institute of Gastroenterology, Yonsei University College of Medicine, Seoul, Republic of Korea

4 Yonsei Liver Center, Severance Hospital, Seoul, Republic of Korea
Various risk factors for NAFLD have been identified, such as a sedentary lifestyle, poor diet, certain genetic profiles, older age, male gender, and metabolic risk factors, including diabetes and obesity [2]. Recently, many investigators have also shown that skeletal muscle mass has a significant association with NAFLD. Sarcopenia is significantly associated with an increased risk of NAFLD among the general population, and of significant liver fibrosis among subjects with NAFLD [3]. In the current issue of Hepatology International, Cai et al. performed a meta-analysis of 19 studies [4] and found that the skeletal muscle index (SMI) in subjects with NAFLD was 1.77 times [95\% confidence interval (CI) 1.15-2.39] lower than that in normal controls. They also reported significantly increased risks of NAFLD [odds ratio (OR) 1.33; 95\% CI 1.20-1.48], NASH (OR 2.42; 95\% CI 1.27-3.57), and NAFLD-related significant fibrosis (OR 1.56 ; 95\% CI 1.34-1.78) in sarcopenic individuals compared with non-sarcopenic subjects. This meta-analysis extensively included cross-sectional and longitudinal studies with further investigation of trim-and-fill test to evaluate the effect of publication bias, and also conducted subgroup analyses according to the methodology of SMI assessment and severity of NAFLD. Although this meta-analysis confirmed the significant association between sarcopenia and NAFLD, several issues should be considered when interpreting the results.

First, although the authors reproduced their results in various subgroups, the tools used to assess skeletal muscle mass (dual-energy X-ray absorptiometry, bioimpedance analysis, or computed tomography), fibrotic burden (liver biopsy or noninvasive surrogates including the fibrosis- 4 index and NAFLD fibrosis score), and steatotic burden (liver biopsy or noninvasive surrogates including ultrasound, the hepatic steatosis index, and the fatty liver index) differed among the included studies, which might lead to marked discordance in definitions of sarcopenia, significant fibrosis, and NAFLD [5].

Second, the definition of sarcopenia is being changed to focus more on the functional status of skeletal muscle, 
rather than just the absolute amount. The recent consensus statement of the European Working Group on Sarcopenia in Older People (EWGSOP) recommends considering 'low muscle strength' and 'low muscle quality' in the definition of sarcopenia. Although most studies that used skeletal muscle mass alone to define sarcopenia reported poor prognostic value of low skeletal muscle mass, future studies should include the strength and quality of skeletal muscle in the diagnostic criteria for sarcopenia, to enable more accurate prognostication within the group with low skeletal muscle mass.

Third, there is no operational definition of absolute skeletal muscle mass. Historically, the appendicular skeletal muscle mass was adjusted by height squared to evaluate low muscle mass, and this approach was recently endorsed by the EWGSOP. However, the US-based Foundation for the National Institutes of Health Sarcopenia Project recommended adjusting the appendicular lean mass using the body mass index to obtain the low lean mass [6]. Several studies, including those in this meta-analysis, have adopted appendicular skeletal muscle mass adjusted using body weight as an index of low muscle mass. Depending on the definition of sarcopenia, an inverse association may be observed between NAFLD and sarcopenia, which should be investigated in studies including detailed methodologies and representative study populations. When sarcopenia is defined based on body weight or body mass index, healthy obese individuals are likely to be categorized as sarcopenic, resulting in biased results. As obesity is the single greatest risk factor for NAFLD, stringent statistical analysis is needed to exclude any influence of obesity from the association between sarcopenia and NAFLD, such as stratified analysis, although the meta-analysis by Cai et al. could not conduct this subgroup analysis due to insufficient data. Supporting this point, two recent retrospective cohort studies showed that the incidence of NAFLD was significantly higher among individuals with sarcopenia, and among those losing lean mass, while this association disappeared in the obese population [7, 8].

Fourth, to understand the cross-sectional, epidemiological association between sarcopenia and NAFLD, we should seek to determine the underlying mechanism. Besides obesity, insulin resistance, chronic inflammation, and lipotoxicity promote the development of both disorders. Skeletal muscle is both a metabolic and an endocrine organ and secretes various substances such as myokines that can mediate crosstalk with the liver [9]. Of these, interleukin-6 and inhibition of myostatin played protective roles against the development of hepatic steatosis in mice, while irisin, an exercise-induced myokine, was negatively associated with the severity of fatty liver in obese humans. This evidence supports the hypothesis that muscle plays a causative role in NAFLD, but more direct evidence is required from future human studies. It has also been proposed that NAFLD plays a role in reducing skeletal muscle mass [10].

Fifth, this meta-analysis did not touch the up-to-date issue of myosteatosis and sarcopenic obesity, due to insufficient information in the included respective studies. Because recent studies have shown that sarcopenic obesity and myosteatosis which is related to the quality or function of skeletal muscle, are associated with the prognosis of patients with chronic liver diseases, further investigations are needed to clarify complex links among skeletal muscle, fat, and liver.

Finally, in addition to this meta-analysis showing a crosssectional association between sarcopenia and NAFLD, further longitudinal studies are required to determine the prognostic implications of sarcopenia in the general population, especially for patients with NAFLD. Recently, sarcopenia and NAFLD have been recognized as risk factors for cardiovascular disease, cerebrovascular disease, type 2 diabetes, and chronic kidney disease [11]. Based on the strong relationship between sarcopenia and NAFLD, studies should investigate whether sarcopenia and NAFLD have an additive or synergistic effect on cardiometabolic and renal diseases. Particularly, a recent study showed that the most important prognostic factor in patients with NAFLD is the fibrotic burden, not inflammatory activity or simple steatotic burden [12], and most patients with NAFLD have simple steatosis without progressive fibrosis or necroinflammation and present favorable long-term prognosis. Therefore, the findings from studies on patients with NAFLD, which can be significantly influenced by the diverse proportion of enrolled patients with NASH, should be interpreted with caution.

In conclusion, the paper by Cai et al. in this issue of Hepatology International makes an important contribution to the literature of NAFLD. Their data provide more evidence that sarcopenia is a predictor or cause of hepatic steatosis and fibrosis. Further prospective studies should focus on the pressing medical issue of whether a change in skeletal muscle function effected through therapeutic intervention can change the course of NAFLD.

Funding This study was supported by Basic Science Research Program through the National Research Foundation of Korea (NRF) funded by the Ministry of Science, ICT \& Future Planning (2019R1A2C4070136) and the grant from the Korea Healthcare Technology R\&D Project, Ministry of Health and Welfare, Republic of Korea (HI17C0913). The funders had no role in study design, data collection and analysis, decision to publish, or preparation of the manuscript.

\section{Compliance with ethical standards}

Conflict of interest Nothing to declare for Yong-ho Lee, Seung Up Kim. 


\section{References}

1. Kim D, Li AA, Gadiparthi C, Khan MA, Cholankeril G, Glenn JS, Ahmed A. Changing trends in etiology-based annual mortality from chronic liver disease, from 2007 through 2016. Gastroenterology. 2018. https://doi.org/10.1053/j.gastro.2018.07.008.

2. Fan JG, Kim SU, Wong VW. New trends on obesity and NAFLD in Asia. J Hepatol. 2017;67(4):862-73.

3. Lee YH, Kim SU, Song K, Park JY, Kim DY, Ahn SH, Lee BW, Kang ES, Cha BS, Han KH. Sarcopenia is associated with significant liver fibrosis independently of obesity and insulin resistance in nonalcoholic fatty liver disease: Nationwide surveys (KNHANES 2008-2011). Hepatology. 2016;63(3):776-86.

4. Cai C, Song X, Chen Y, Chen X, Yu C. Relationship between relative skeletal muscle mass and nonalcoholic fatty liver disease: a systematic review and meta-analysis. Hepatol Int. 2019. https:// doi.org/10.1007/s12072-019-09964-1.

5. Jo MH, Lim TS, Jeon MY, Lee HW, Kim BK, Park JY, Kim DY, Ahn SH, Han KH, Kim SU. Predictors of discordance in the assessment of skeletal muscle mass between computed tomography and bioimpedance analysis. J Clin Med. 2019;8(3):E322. https://doi.org/10.3390/jcm8030322.

6. Studenski SA, Peters KW, Alley DE, Cawthon PM, McLean RR, Harris TB, Ferrucci L, Guralnik JM, Fragala MS, Kenny AM, Kiel DP, Kritchevsky SB, Shardell MD, Dam TT, Vassileva MT. The FNIH sarcopenia project: rationale, study description, conference recommendations, and final estimates. J Gerontol A Biol Sci Med Sci. 2014;69(5):547-58.

7. Kim G, Lee SE, Lee YB, Jun JE, Ahn J, Bae JC, Jin SM, Hur KY, Jee JH, Lee MK, Kim JH. Relationship between relative skeletal muscle mass and nonalcoholic fatty liver disease: a 7-year longitudinal study. Hepatology. 2018;68(5):1755-68.

8. Lee MJ, Kim EH, Bae SJ, Kim GA, Park SW, Choe J, Jung CH, Lee WJ, Kim HK. Age-related decrease in skeletal muscle mass is an independent risk factor for incident nonalcoholic fatty liver disease: a 10-year retrospective cohort study. Gut Liver. 2019;13(1):67-76.

9. Pedersen BK, Febbraio MA. Muscles, exercise and obesity: skeletal muscle as a secretory organ. Nat Rev Endocrinol. 2012;8:457-65.

10. Bhanji RA, Narayanan P, Allen AM, Malhi H, Watt KD. Sarcopenia in hiding: the risk and consequence of underestimating muscle dysfunction in nonalcoholic steatohepatitis. Hepatology. 2017;66(6):2055-65.

11. Han E, Lee YH. Non-alcoholic fatty liver disease: the emerging burden in cardiometabolic and renal diseases. Diabetes Metab J. 2017;41(6):430-7.

12. Dulai PS, Singh S, Patel J, Soni M, Prokop LJ, Younossi Z, Sebastiani G, Ekstedt M, Hagstrom H, Nasr P, Stal P, Wong VW, Kechagias S, Hultcrantz R, Loomba R. Increased risk of mortality by fibrosis stage in nonalcoholic fatty liver disease: systematic review and meta-analysis. Hepatology. 2017;65(5):1557-65.

Publisher's Note Springer Nature remains neutral with regard to jurisdictional claims in published maps and institutional affiliations. 